\title{
Les institutions spécialisées de riposte contre le covid-19 et la promotion de l'état de droit en RDC : état des lieux, défis et perspectives
}

\author{
Par Moise ABDOU MUHIMA*
}

\section{Résumé}

La riposte pour contenir la menace dû à la pandémie de Covid-19 a exigé de prendre certaines mesures et dispositions radicales allant jusqu'à la proclamation de l'état d'urgence sanitaire pour ralentir ou stopper la propagation de la pandémie en vue de protéger la santé des populations en République Démocratique du Congo (RDC). Dans la foulée, le pouvoir public ne s'est pas contenté des institutions existantes pour faire face à cette pandémie. Ainsi plusieurs autres institutions spécialisées ont vu le jour pour intervenir dans divers secteurs de la riposte contre le coronavirus. Cette étude a relevé principalement la Cellule de riposte contre le Covid 19 en RDC; la Task force présidentielle; le Secrétariat technique; le Fonds national de solidarité contre le Coronavirus et le Comité multisectoriel de riposte (CMR-COVID-19). La quasi-totalité de toutes ces institutions sont rattachées directement à la Présidence de la République et rendent compte directement au Président de la République et leurs animateurs au plus haut niveau sont pour la plupart des personnels de santé qui du reste veillent moins aux questions de l'Etat de droit. Il est fustigé dans cet article le risque de dédoublement institutionnel causé par cette inflation institutionnelle qui ne garantie pas la promotion et la protection des valeurs et principes de l'Etat de droit en RDC et que la solution résiderait dans une limitation strict de la création des nouvelles institutions tout en s'appuyant sur les institutions existantes, quitte aux pouvoirs publics de veiller simplement à bien les équipées et leurs donner des missions claires et spécifiques.

\begin{abstract}
The response to contain the threat arising from Covid-19 pandemic required taking certain drastic measures and steps up to the declaration of a state of health emergency to slow down or stop the spread of the pandemic in order to protect the health of the population in Congo. In the meantime, the public authorities did not limit themselves to the existing institutions to deal with the pandemic. Thus, several other specialized institutions were created to intervene in various sectors of the response to the coronavirus. The main ones identified in this study were «la Cellule de riposte contre le Covid 19 en RDC; la Task force prési-

* Licencié en Droit, Faculté de Droit de l'Université de Kinshasa (R.D Congo); Master Cross-border E-commerce, Zhejiang GongShang University (Hangzhou-China) et Avocat au Barreau de Kinshasa / Matete. E-mail : muhimamoise@gmail.com.
\end{abstract}


dentielle; le Secrétariat technique; le Fonds national de solidarité contre le Coronavirus et le Comité multisectoriel de riposte (CMR-COVID-19) ». Almost all of these institutions are directly attached to the Presidency of the Republic and are accountable directly to the President of the Republic, and their leaders at the highest level are for the majority healthcare personnel, who moreover are less attentive to questions of the rule of law. This article criticizes the risk of institutional duplication caused by this institutional inflation, which does not guarantee the promotion and protection of the values and principles of the rule of law in Congo and the solution would lie in strictly limiting the creation of new institutions while relying on existing institutions, upon the public authorities to simply ensure that they are well equipped and give them clear and specific missions.

\section{Introduction}

La pandémie de Covid-19 a secoué toutes les nations du monde sans exception aucune. Alors que le Covid-19 se propage sur le continent africain, les dirigeants de la RDC y ont rapidement réagi en déployant tous les moyens nécessaires pour y faire face. Ces dirigeants représentant le nouveau régime ont faits de la promotion des valeurs démocratiques, du respect et de la protection des droits de l'homme, de la lutte contre la corruption leur cheval de batail. De l'autre cote, la riposte pour contenir la menace dû au Covid-19 a exigé de prendre certaines mesures et dispositions radicales allant jusqu'à déclarer même l'état d'urgence pour ralentir ou stopper la propagation de la pandémie et protéger la santé des populations. Les mesures de ce type ont, pour la plupart, d'importantes répercussions sur l'exercice des droits de l'homme et plus encore, l'effectivité de l'Etat de droit.

L'exercice intellectuel et matériel pour apprécier à juste titre l'opportunité et la proportionnalité de ces mesures restrictives face aux valeurs et principes de l'Etat de droit, notamment la protection des droits de l'homme, demeure sujette à controverses des points de vue. Tout de même, la limitation doit être appropriée, c'est-à-dire qu'elle doit raisonnablement avoir un effet de protection de la sante publique dans le contexte de la pandémie de Covid-19 et elle doit représenter le moyen le moins intrusif parmi tous les moyens susceptibles d'atteindre cet objectif ${ }^{1}$. Mais aussi, les avantages de la limitation doivent l'emporter sur ses inconvénients. Ainsi, il apparait vraisemblable que la pandémie de Covid-19 met à l'épreuve les valeurs fondamentales des sociétés, des gouvernements, des communautés et des individus ${ }^{2}$ à telle enseigne qu'a un certain moment l'heure était à la solidarité et à la coopération afin de lutter contre le virus et d'atténuer ses effets, souvent imprévus, des mesures visant à stopper la propagation du virus. A l'approche dupic de la pandémie, les différents acteurs semblaient être d'accord avec n'importe quelle mesure à condition d'être épargner de la catastrophe irréversible en s'abandonnant entièrement entre les mains des spécia-

1 Louis FAVOREU at al., Droit constitutionnel, 21ème Édition, Paris, 2019, p.217.

2 Kambale Kambale Pascal, Impact de la crise de covid-19 sur le processus démocratique en RDC, disponible sur http://congoresearchgroup.org/impact-de-la-crise-de-covid-19-sur-le-processus-demo cratique-en-rdc/?lang=fr. 
listes de la riposte aux cotés des autorités gouvernementales. A ce stade, les défenseurs des droits de l'homme les plus acharnés voire même les juges ou les parlementaires qui constituent le rempart institutionnels étatiques pour la protection des valeurs et principes de l'Etat de droit, notamment la protection des droits de l'homme face à l'Exécutif super puissant. Ainsi en ce qui concerne les droits de l'homme, on serait en droit de se poser la question de savoir « qui défendra le défenseur des droits de l'homme? » Ou du moins, « qui protégera le protecteur des droits de l'homme?».

L'Exécutif en RDC a fait recours à des institutions et des personnalités spécialisées puisque lui-même ne disposant pas des compétences nécessaires en matière de riposte contre une telle pandémie. Pour la majorité d'entre ces spécialistes, ils sont médecins spécialistes virologues ou autres, ou encore généralistes ou infirmiers. Repartis dans divers institutions, ces médecins et infirmiers ont jusqu'à ce jour le pouvoir d'influencer ou d'orienter les décisions de l'Exécutif en ce qui concerne les mesures à prendre pour faire face à la pandémie de Covid-19. Et une anecdote naissante dans la ville de Kinshasa confirmait que « Muyembe avait le pouvoir de confiner et de déconfiner! ». Comment les normes de base en matière de l'Etat de droit peuvent-elles guider les efforts que font ces spécialistes de santé pour agir face à la crise sanitaire? Comment veiller à ce que les actions du gouvernement soient compatibles avec leurs obligations en matière de promotion et de protection des valeurs et principes de l'Etat de droit? Quel est le juste équilibre entre le respect de ces valeurs et principes et l'atténuation des effets indésirables des mesures de confinement, de l'Etat d'urgence ou encore du couvre-feu sur la promotion de l'Etat de droit tant vénéré par le régime en place? Ainsi, le plus grand défi est que le cadre légal et institutionnel dans lesquels évoluent ces « spécialistes de santé» volant au secours de l'Exécutif et des services de santé traditionnels doit être méticuleusement établis pour éviter à ce qu'ils ne sacrifient pas les valeurs les plus fondamentales de l'Etat de droit en RDC.

\section{A. Cellule de riposte contre le Covid-19 en RDC et l'Equipe de la task force présidentielle}

Au sommet de la structure institutionnelle de la riposte contre le Covid-19 en RDC où se trouve le Président de la République ${ }^{3}$, il est directement appuyé par deux institutions spécialisées qui toutes sont directement attaché à la Présidence de la République et rendent directement des comptes au Président de la République. Il s'agit de la Cellule de riposte contre le Covid 19 en RDC et la Task force présidentielle. La Cellule de riposte contre le Covid-19 en RDC est dotée d'une autonomie administrative et technique, fonctionnant sous l'autorité du Président de la République ayant pour principale mission d'éclairer les choix de l'Exécutif dans la gestion de la crise sanitaire provoquée par le Covid-19; de lui donner

3 RDC, Plan de préparation et de riposte contre l'épidémie au Covid-19 en République Démocratique du Congo, Secrétariat technique de la riposte, mars 2020, p. 9. 
des avis sur les mesures prises en vertu de la situation de l'urgence ${ }^{4}$. La Coordination de la cellule de Riposte contre le Covid-19 est dirigé par le professeur docteur Jean-Jacques MUYEMBE TANFU ${ }^{5}$. Aux côtés de la Cellule de riposte contre le Covid-19, le Président de la République a créé une autre institution spécialisée en date du 02 mai 2020 dénommée Task force présidentielle qui fonctionne sous son autorité directe. Cette Task force a pour mission d'assister le Président de la République dans le suivi, la supervision et l'évaluation de toutes les activités de prévention et de la riposte contre la pandémie de Coronavirus ou d'autres pandémie, épidémie ou maladies à virus en $\mathrm{RDC}^{6}$.

Aux fins d'éviter la propagation de l'épidémie et de garantir la santé publique, le Président de la République a ordonné, sous la coordination de la Cellule de riposte contre le Covid-19, des mesures pour le contrôle sanitaire systématique de toute personne entrant sur le territoire national et de celle au départ des villes, en particulier de la capitale vers les autres provinces du pays. La cellule de riposte contre le Covid-19 veuille à ce que les postes d'entrées maritime, fluviale, lacustre et terrestres du territoire national soient dotés du même dispositif de surveillance pour renforcer le contrôle des passagers en provenance de l'étranger. La mise en quarantaine des personnes présentant les symptômes de Covid-19 et susceptibles d'être affectées par le Coronavirus et le placement et le maintien en isolement, à leur domicile ou tout autre lieu d'hébergement adapté, des personnes affectées par la pandémie.

A la suite des recommandations de la Cellule de riposte, le Président de la République a instruit le gouvernement de trouver les moyens de ravitailler les agglomérations à grande affluence où le manque d'eau et d'électricité est quasi permanent, pour assurer l'hygiène et de multiplier les points de lavage des mains dans les aéroports du pays et appuyer les équipes de la Régie des voies aériennes (RVA) et de la Direction générale des Migrations (DGM) pour que nul n'échappe au contrôle d'hygiène.

La Task force présidentielle est une Commission ad hoc dont la durée est liée à l'éradication de l'épidémie du Covid-19 en RDC. Mais, le Président de la République pourra souverainement décider de proroger la durée de la Task force s'il l'estime nécessaire ${ }^{7}$. Dans la riposte contre le Covid-19, la Task force propose au Président de la République les orientations et les mesures spécifiques multisectorielles de la prévention et de la riposte. Elle participe à la mobilisation des moyens tant matériels que financiers à l'échelle nationale et internationale pour endiguer ces fléaux et suit la gestion des mesures arrêtées par le Gouvernement en collaboration avec les différentes autorités et institutions intervenant dans la lutte contre l'épidémie de Covid-19 ou d'autres pandémies, épidémies ou maladies à virus. Cette

4 Article $4 \S 1$ de l'Ordonnance n²0/014 du 24 mars 2020 portant proclamation de l'état d'urgence sanitaire pour faire face à l'épidémie de Covid- 19 .

5 Article $04 \S 2$ de $1^{\prime}$ Ordonnance $\mathrm{n}^{\circ} 20 / 014$ du 24 mars 2020 note 3.

6 Article 2 de l'Ordonnance n²0/036 du 02 mai 2020 portant création, organisation et fonctionnement d'une Commission ad hoc dénommée « Task force présidentielle.

7 Article $1^{\text {er }}$ de $1^{\prime}$ Ordonnance $n^{\circ} 20 / 036$ du 02 mai 2020, note 5. 
Commission évalue les conséquences politiques, socio-économiques, sécuritaires et diverses de la pandémie ou tout autre maladies à virus et propose au Président de la République des mesures idoines dans les meilleurs délais et lui faire rapport sur l'évolution de la prévention et de la riposte contre la pandémie de Covid-19 ou de tout autre pandémie, épidémie ou maladies à virus sur le territoire national ${ }^{8}$.

La Task force présidentielle est dirigée par un bureau composé d'un coordonnateur, d'un Coordonateur adjoint, d'un Rapporteur et d'une Rapporteur adjoint. Elle se réunit au moins une fois par semaine et chaque fois que le besoin se présente à la demande de la majorité de ses membres ou du Président de la République à qui elle rend compte de ses activités au cours des séances de restitutions ${ }^{9}$. En dehors de ses membres, la Task force peut recourir à l'expertise de toute personne dont les compétences techniques et professionnelles peuvent contribuer à la réalisation de sa mission ${ }^{10}$. Ainsi, avec la possibilité de recourir à l'expertise extérieure, il apparait évident que la Task force Présidentielle n'est pas uniquement composée des personnes ayant une expertise dans le domaine médical ou de la santé publique, et en plus, elle comprend même des membres faisant partie du Cabinet du Président de la République ${ }^{11}$.

\section{B. Comité Multisectoriel de Riposte contre la COVID-19 en RD}

Le Comité multisectoriel de riposte (CMR-COVID-19) est présidé par le Premier ministre, le Ministre de la Santé assurant le secrétariat. Il donne les orientations politiques du gouvernement, mobilise les ressources et gère les fonds. Le CMR-COVID-19 comprend principalement les ministères suivants : Intérieur, sécurité et affaires coutumières; Santé; Finances; Budget; Communication et médias; Transport et voies de communication; Pêche et élevage; Plan; Affaires Sociales; Actions Humanitaires et solidarité nationale; Enseignement supérieur et universitaire; Recherche scientifique et Innovation technologique; Défense nationale et anciens combattants; Justice et garde des sceaux; Enseignement primaire, secondaire et technique; Environnement et Développement Durable; Coopération Internationale, Intégration régionale et francophonie; Affaires Etrangères. A côté des représentants des différents ministères, le Comité multisectoriel de riposte comprend un Secrétariat technique et tous, leurs activités sont soutenues par un fonds national de solidarité pour la riposte contre le Covid-19 en RDC.

Le Secrétariat technique est dirigé par un coordonnateur nommé par le Président de la République. Le Secrétaire Général à la Santé, l'Inspecteur Général de la Santé, les représentants des partenaires techniques et financiers (principalement co-lead), les représentants

8 Article 2 de l'Ordonnance $\mathrm{n}^{\circ} 20 / 036$ du 02 mai 2020, note 5.

9 Article 6 de l'Ordonnance $n^{\circ} 20 / 036$ du 02 mai 2020, note 5.

10 Article 7 de l'Ordonnance n²0/036 du 02 mai 2020, note 5.

11 Article $1^{\text {er }}$ de l'Ordonnance $\mathrm{n}^{\circ} 20 / 037$ du 02 mai 2020 portant nomination des membres de la Task force présidentielle. 
de la société civile ainsi que toute autre personne sollicitée par le Coordonnateur du Secrétariat technique constituent le Conseil consultatif du Secrétariat technique. Le Secrétariat technique assure la gestion stratégique de toutes les commissions de riposte et de préparation. Il est soutenu par un Incident Manager (du niveau national) et un Incident Manager adjoint (Chef de Division Provinciale de la Santé de la Province affectée/concernée) pour coordonner toutes les interventions de la riposte et de la préparation. Les instances rattachées au secrétariat technique et regroupant les techniciens autour de différentes thématiques appuient l'Incident Manager et son adjoint à travers divers sections.

La première section est chargée des Opérations de Réponse, autour des commissions surveillance, prise en charge médicale, prise en charge psycho-sociale, communication des risques et engagement communautaire, Prévention et Contrôle de l'Infection, Laboratoire et recherche. L'autre section concerne les opérations de Préparation comprenant le renforcement des piliers avec les différentes thématiques traditionnelles, les équipes d'intervention rapide, les exercices de simulation. L'Appui au Système de Gestion de l'Information comprend la gestion de l'information et des données, planification, suivi et évaluation, soutien aux COUSP. La Logistique, Services d'appui et Mitigation des risques comprend la Commission Logistique, l'Administration, les Finances, Commission Suivi des mesures de Mitigation des risques et Distanciation Sociale, y compris Sécurité.

Le Fonds national de solidarité contre le Coronavirus a été crée par Ordonnance du Président de la République $\mathrm{n}^{\circ} 20-018$ du 6 avril $2020^{12}$. Ce Fonds a pour principale mission de rechercher et collecter des moyens financiers destinés à servir, sous forme d'aides, assistance ou soutiens, aux personnes physiques ou morales, personnels médicaux soignants, services médicaux et hospitaliers ainsi qu'aux entreprises et autres structures exerçant une activité économique, qui seraient particulièrement touchés par les conséquences économiques, financières et sociales de la propagation du Covid-19 et des mesures prises pour en limiter la propagation ${ }^{13}$. Il est alimenté par l'État et, sur une base volontaire, par les provinces, les entités territoriales décentralisées, les entreprises publiques ou privées, les bailleurs de fonds, les partenaires et toute autre personne physique ou morale de droit public ou privé. Les moyens financiers récoltés et mis à la disposition du Fonds sont logés dans un compte spécial qui sera ouvert par le coordonnateur du comité de gestion du Fonds dans une des banques commerciales ${ }^{14}$.

Le fonds est destiné notamment à financer les fournitures essentielles comme les équipements de protection individuelle à la population et aux agents de santé qui sont en première ligne sur l'étendue du territoire national; à soutenir financièrement le comité multisectoriel de riposte au Covid-19 en lui donnant les moyens pour suivre et détecter la maladie et en renforçant les capacités de laboratoire par la formation et la fourniture de matériels

12 Article $1^{\text {er }}$ de l'Ordonnance $\mathrm{n}^{\circ} 20-018$ du 6 avril 2020 Portant création, organisation et fonctionnements du Fonds national de solidarité contre le Coronavirus en RDC.

13 Article 2 de l'Ordonnance $n^{\circ} 20-018$ du 6 avril 2020, note 11.

14 Article 4 de l'Ordonnance $n^{\circ} 20-018$ du 6 avril 2020, note 11. 
adéquats. Ce fonds veille à soutenir financièrement les agents de santé et les communautés locales pour qu'ils aient partout accès aux dernières informations scientifiques pour pouvoir se protéger, prévenir l'infection, endiguer sa propagation et dispenser des soins à ceux qui ont besoin de manière à atténuer l'impact social du Covid-19, en particulier sur les femmes, les enfants et les groupes sociaux vulnérables et financer l'intensification des efforts visant à accélérer l'approvisionnement tant en produits de diagnostic et traitements permettant de sauver des vies qu'en biens et denrées de première nécessité. Aussi, il sert à soutenir financièrement les entreprises uniquement en difficultés en raison de l'épidémie du Covid-19 et toutes initiatives destinées à éviter de perturber la chaîne d'approvisionnement alimentaire aux conséquences prononcées pour la population.

La gestion du Fonds et la réalisation de ses missions sont confiées à un Comité de gestion composé d'au moins sept Experts dont un Coordonnateur, un Coordonnateur Adjoint, un délégué de la Société civile, un délégué des Mouvements associatifs des Femme, un délégué des entreprises du secteur public un délégué des entreprises du secteur privé ainsi que le Secrétaire Technique du Comité Multisectoriel de Riposte au Covid-19. Toutes ces personnalités doivent jouir d'une crédibilité et moralité sans faille et travailler sous l'autorité et la supervision du Président de la République. Et Pour garantir la transparence dans le gestion, la traçabilité des opérations d'assistance et un contrôle de toutes les donations centralisées et destinées à la riposte sur l'étendue du territoire national, un auditeur externe choisi parmi les membres de l'Ordre des experts comptables justifiant d'une expérience et d'une compétence avérées et de très bonne réputation sera désigné par le président de la République pour certifier dans les conditions fixées par la réglementation en vigueur les comptes du Fonds établis par le comité de gestion avant leur présentation au chef de l'État.

\section{Conclusion}

En réponse à la pandémie de COVID-19, plusieurs états du monde, notamment la RDC ont eu recours à des outils permettant de suivre et de contrôler le comportement et les mouvements des individus. Afin de satisfaire aux critères de nécessité et de proportionnalité, ces outils doivent être spécifiquement liés à des objectifs de santé publique, être utilisés exclusivement à cette fin, et avoir une durée et une portée limitées selon ce qu'exige la situation. Si des restrictions à la liberté de mouvement peuvent se justifier pour arrêter la propagation du virus, il peut, en revanche, s'avérer très difficile de justifier des limitations de certains autres droits humains.

En RDC, le pouvoir Exécutif a pris des mesures qui ont créées en même temps plusieurs institutions spécialisées pour le besoin de la cause. Ces institutions spécialisées ont pour préoccupation principale de contenir et contrôler la menace du à la pandémie de Covid-19 sur l'ensemble du territoire national, cela implique un grand nombre de la population et plusieurs paramètres à analyser chaque fois qu'une décision doit être prise. Etant pour la plupart des professionnel de santé, l'enjeu devient très important puisqu'ils peuvent mal utiliser les prérogatives énormes qui leurs sont confiées pendant cette période de l'Etat 
d'urgence au risque de ne pas tenir compte de l'impératif de respect et de protection des droits de l'homme. Ce n'est pas parce qu'il faut lutter contre la pandémie de Covid-19 que les droits de l'homme doivent être sacrifiés à tout bout de champ.

\section{Bibliographie}

\section{Textes juridiques}

Arrêté ministériel No. 25/CAB/VPM/ MININTERSECAC/GKM/06/2020 du 25 mars 2020 portant modalités pratiques d'exécution des mesures de limitation de la propagation de la pandémie de Covid-19

Constitution de la RDC du 18 février 2006, in J.O.R.D.C, 47ème année, numéro spécial du 18 février 2006 telle que modifiée en 2011.

Ordonnance n $20 / 029$ du 23 avril 2020 portant mesures complémentaires nécessaires pour faire face à l'épidémie au Covid 19

Ordonnance n`20/033 du 02 mai 2020 modifiant et complétant l'ordonnance n²0/018 du 06 avril 2020 portant création, organisation et fonctionnement d'un Fonds national de solidarité contre le Coronavirus, en sigle « FNSCC »

Ordonnance $n^{\circ} 20 / 036$ du 02 mai 2020 portant création, organisation et fonctionnement d'une commission ad hoc dénommée « Task Force Présidentielle».

Ordonnance n ${ }^{\circ} 20-018$ du 6 avril 2020 portant création, organisation et fonctionnement d'un Fonds national de solidarité contre le Coronavirus, en sigle « FNSCC

Ordonnance numéro 20/014 du 24 mars 2020 portant proclamation de l'état d'urgence sanitaire pour faire face à la pandémie de Covid-19

Pacte international relatif aux droits économiques, sociaux et culturels («PIDESC ») du 16 décembre 1966

\section{Ouvrages}

Louis FAVOREU at al., Droit constitutionnel, 21ème Édition, Paris, 2019

Institute for human rights and development in Africa, Décisions de la Commission africaine des droits de l'homme et des peuples sur les Communications, 2002-2007, Cape Town, Institue for Human Rights and Development in Africa, 2008.

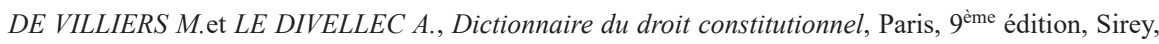
2013.

ILUME MOKE Michel, Droit constitutionnel et Institutions politiques de la République Démocratique du Congo, Kisangani, 2018. 


\section{Articles}

KAMBALE KAMBALE Pascal, Impact de la crise de covid-19 sur le processus démocratique en RDC, disponible sur http://congoresearchgroup.org/impact-de-la-crise-de-covid-19-sur-le-processus-dem ocratique-en-rdc/?lang $=\mathrm{fr}$

BUJAKERA TSHIAMALA Stanis, «En RDC, l'état d'urgence divise la coalition au pouvoir », https://w ww.jeuneafrique.com/927819/politique/en-rdc-letat-durgence-divise-la-coalition-au-pouvoir/, (Consulté le 12/01/2021).

GATA-KAMBUDI Aimé, « La constitutionnalité ou non de l'ordonnance présidentielle proclamant l'état d'urgence en RDC : qui dit vrai? », https://desc-wondo.org/la-constitutionnalite-ou-non-de-lordon nance-presidentielle-proclamant-letat-durgence-en-rdc-qui-dit-vrai-aime-gata-kambudi/, (Consulté le $15 / 09 / 2020)$

\section{Rapports et Autres documents}

$R D C$, Plan de préparation et de riposte contre l'épidémie au Covid-19 en République Démocratique du Congo, Secrétariat technique de la riposte, mars 2020

Commission africaine des droits de l'homme et des peuples, Documentation, $\mathrm{n}^{\circ} 1$, Banjul.

Haut Commissariat des Nations Unies aux droits de l'homme, « Emergency measures and covid-19 